\title{
Konfessionslosigkeit und Globalisierung: Eine neue Herausforderung für die religionspädagogische Forschung
}

\begin{abstract}
Zusammenfassung
Es gibt eine wachsende Zahl an Forschungsarbeiten, die sich mit dem Zusammenhang von Globalisierung, Religion und Bildung beschäftigen. Der vorliegende Artikel bezieht diese Arbeiten auf den Religionsunterricht an öffentlichen Schulen in Deutschland. Diesen besuchen nicht nur Kinder und Jugendliche aus unterschiedlichen Konfessionen und Religionen, sondern auch Konfessionslose. Ausgehend von dieser Beobachtung wird zunächst die Pfadabhängigkeit religiöser Entwicklungen in einem globalen Kontext beschrieben, indem Ostdeutschland als Fallbeispiel herangezogen wird. Anschließend werden die nationalen, konfessionellen und religiösen Implikationen skizziert, die mit Begriffen wie „konfessionslos“, „bekenntnisfrei" und "nicht-religiös“ einhergehen. Abschließend werden die schulischen und außerschulischen Aufgaben religionsbezogener Bildung in einer globalisierten Welt erkundet.
\end{abstract}

Schlüsselworte: transnationale Bildungsforschung, religionsbezogene Bildung, Ostdeutschland

\begin{abstract}
There is a growing body of literature that recognizes the interplay of globalization, religion, and education. This article applies these literatures to Religious Education (RE) at public schools in Germany. RE is attended not only by children and by adolescents of different denominations and religions but also by those with no religious affiliation. Starting from this observation, the article considers the path dependency in the history of religion in a global context, taking East Germany as an example. Secondly, the article takes into account the different national, confessional, and religious epistemologies that frame the terminology ",non-denominational”, „non-confessional", and "non-religious". Finally, the article explores teaching concepts for religious education in public schools and extracurricular learning.
\end{abstract}

Keywords: transnational Education, religious Education, East Germany

\section{Einführung}

Schülerinnen und Schüler, die sich auf der Homepage einer Austauschorganisation für ein Schuljahr in den USA interessieren, können dort unter anderem den folgenden, eindringlichen Hinweis lesen: „Du solltest bereit sein, zusammen mit deiner Gastfamilie am Kirchenbesuch teilzunehmen. Die Familie möchte dich schließlich in ihren Alltag und ihre Kultur integrieren. Dazu gehört oftmals auch die Ausübung der Religion und du solltest dich diesem Teil des Familienlebens nicht von vornherein verschließen" (GLS Sprachenzentrum Berlin, 2020).

Auch wenn das Bildungsangebot der Austauschorganisation nicht zwischen konfessionsgebundenen und konfessionslosen Schülerinnen und Schülern differenziert, wird deutlich, dass nicht nur das interkonfessionelle und interreligiöse Lernen, sondern auch das gemeinsame Lernen mit Konfessionslosen (als Teilbereich interkultureller Bildung im Rahmen einer erziehungswissenschaftlichen Theorie der Weltgesellschaft und Globalisierung) eine zentrale Herausforderung für die pädagogische Forschung ist (zum Verhältnis dieser Lerndimensionen vgl. Asbrand \& Scheunpflug, 2005). Auch auf der Seite der Konfessionslosen wächst mit dem Wissen über globale Zusammenhänge, aber auch durch private Urlaubsreisen, berufliche Mobilität und schulische Austauschprogramm das Wissen über Konfessionen und Religionen, die im regionalen und lokalen Kontext keine Rolle spielen.

Aus dem Füllhorn an Forschungsfragen, die sich aus der Globalisierung aller Lebensbereiche für die Religionspädagogik ergeben, seien im Folgenden einige wenige Aspekte ausgeführt. Zunächst geht es darum, die historische Pfadabhängigkeit gesellschaftlicher Entwicklungen zu verstehen und auf das Phänomen der Konfessionslosigkeit zu beziehen. Die Beschäftigung mit dem Phänomen führt zudem die epistemischen Grenzen der Vergleichbarkeit vor Augen, so dass Fragen nach der sprachlichen und kulturellen Übersetzbarkeit (im Rahmen eines transnationalen Forschungsansatzes) zu bedenken sind. Im letzten Abschnitt geht es schließlich um die sich verändernden Aufgaben religiöser Bildung in einer globalisierten Welt. Alles, was in diesem Abschnitt ausgeführt wird, gilt glei- 
chermaßen für Konfessionslose und Nicht-Konfessionslose. Auf diese Weise wird deutlich: Religiöse Bildung muss ein Teil der Allgemeinbildung für alle Schülerinnen und Schüler sein, ganz gleich, ob diese selbst an einer religiösen Praxis partizipieren oder nicht.

\section{Historische Aspekte: Konfessionslosigkeit und die Pfadabhängigkeit gesellschaftlicher Entwicklungen im globalen Kontext}

Stärker als in älteren Modernisierungstheorien wird heute die historische Pfadabhängigkeit gesellschaftlicher Entwicklungen und religiöser Dynamiken betont. Modernisierungstheorien gingen lange Zeit von einer zeitlich versetzten, im ganzen aber vergleichbaren Pluralisierung, Individualisierung, Privatisierung, Säkularisierung und Ökonomisierung von Religion auf der gesamten Welt aus. Auf das Theorem der Pfadabhängigkeit gestützt (vgl. Käbisch \& Simojoki, 2018) kann demgegenüber betont werden, dass vergangene lokale Kontexte (wie die staatlich forcierte Säkularisierung in der DDR) und frühere Entscheidungen (wie der Kirchenaustritt aus Karrieregründen) spätere Entscheidungen (wie die Partizipation an einer religiösen Praxis) überproportional mitbestimmen, auch wenn frühere Kontexte (wie die 1989 beendete kommunistische Herrschaft in Osteuropa) längst vergangen sind. Um Konfessionslosigkeit als Langzeitphänomen verstehen zu können, müssen daher die lokalen und regionalen Kontexte sowie die Handlungsoptionen der Akteure und deren individuelle Entscheidungen in die Analyse einbezogen werden. Die Verschränkung gesellschafts- und geschichtswissenschaftlicher Zugänge in der Religionspädagogik besitzt daher beim Thema der Konfessionslosigkeit eine hohe Plausibilität.

Angesichts der 1933 beginnenden und bis 1990 auf dem Gebiet der Sowjetischen Besatzungszone (SBZ) bzw. DDR andauernden kirchenfeindlichen Politik der nationalsozialistischen und kommunistischen Herrscher ist gut nachvollziehbar, warum die Kirchenmitgliedschaftsquoten und die Gründe für Konfessionslosigkeit in West- und Ostdeutschland voneinander abweichen. Die individualisierte Distanzierung von Kirche im Sinne eines believing without belonging („Weil ich auch ohne Kirche christlich sein kann") erhält in Westdeutschland eine höhere Zustimmung, in Ostdeutschland ist es hingegen das Normalitätsargument („Weil es in meinem Umfeld normal ist, nicht in der Kirche zu sein"). Kritik an kirchlichen Stellungnahmen werden von einem Drittel der Westdeutschen, jedoch kaum von Ostdeutschen genannt, da kirchliche Stellungnahmen keine Bedeutung für sie haben (Pickel, 2011, S. 58). Eine gleich hohe Zustimmungsrate hat demgegenüber die Kirchensteuer, die in Mitgliedschaftsuntersuchungen der Evangelischen Kirche in Deutschland (EKD) ungefähr von der Hälfte der Konfessionslosen in West und Ost als Grund für einen vergangenen Kirchenaustritt genannt wurde.

Die „diktaturstaatliche Doppelschädigung der mitteldeutschen Kirchen" (Nowak, 1996, S. 40) allein kann die weitgehende Entkirchlichung, die vor allem die evangelische Bevölkerung trifft, jedoch nur zum Teil erklären. Zwei weitere Faktoren werden daher in der Fachliteratur diskutiert: Zum einen die kirchenfeindliche Arbeiterbewegung und organisierte Religionskritik proletarischer Freidenkerverbände in der
Zeit des Kaiserreiches und in der Weimarer Republik, die bereits im ersten Drittel des 20. Jahrhunderts zu einer breiten Kirchenaustrittsbewegung mit alternativen Vergemeinschaftungsformen und Ritualen, wie z.B. der Jugendweihe, führte (vgl. Kaiser, 1981). Zum anderen die im Vergleich zum Katholizismus und zur Orthodoxie geringen Bindungskräfte des Protestantismus, zu dessen Grundüberzeugungen es gehört, dass die Mitgliedschaft in einer Kirche nicht heilsnotwendig sei und die bedingungslose Rechtfertigung auch Menschen einschließt, die aus der Kirche ausgetreten sind oder nie einer Kirche angehört haben. Das Fehlen dieser beiden Faktoren in mehrheitlich römisch-katholischen und orthodoxen Ländern mit ähnlichen Diktaturerfahrungen (z.B. Polen und Russland) erklärt, warum die hohe religiöse Vitalität in diesen Ländern nicht mit der Situation in Ostdeutschland vergleichbar ist (Martin, 2011, S. 149-164).

\section{Transnationale Aspekte: „Konfessionslo- sigkeit" in anderen Ländern und die Grenzen der Vergleichbarkeit}

Die formalen und inhaltlichen Merkmale der Zugehörigkeit bzw. Nicht-Zugehörigkeit von Individuen zur römisch-katholischen oder evangelischen Kirche in Deutschland lassen sich weder auf andere Nationen übertragen noch ohne weiteres in die Mitgliedschaftslogik anderer Konfessionen und Religionen übersetzen. Es gibt auch keinen neutralen Vergleichsstandpunkt, um „Konfessionslosigkeit“ in verschiedenen Ländern, Konfessionen und Religionen beschreiben zu können, da alle Beschreibungskategorien bereits einem bestimmten nationalen, konfessionellen oder religiösen Kontext entstammen. Ausgehend von den Grenzen der Komparatistik plädiert der folgende Abschnitt daher dafür, Fragen der sprachlichen und kulturellen Übersetzbarkeit (im Rahmen eines transnationalen Forschungsansatzes) besondere Aufmerksamkeit zu schenken. Unter formalen Gesichtspunkten betrifft dies zunächst den Umstand, dass Konfessionslosigkeit in Deutschland die Nichtzugehörigkeit zu einer Religionsgemeinschaft als Körperschaft des öffentlichen Rechts meint. Augenfällig wird dieser Umstand u.a. darin, dass Konfessionslose in Deutschland keinen Mitgliedsbeitrag bei einer Kirche zahlen, der über staatliche Finanzämter eingezogen wird und über die Lohnsteuererklärung erstattet werden kann, die sog. Kirchensteuer. Andere Länder wie Frankreich oder England erfassen weder das Verhältnis ihrer Staatsbürger zu bestimmten Religionsgemeinschaften noch erheben sie eine Kirchensteuer, währenddessen beispielsweise in Griechenland alle Bürger der Mehrheitsreligion von Geburt an zugerechnet werden.

Unter inhaltlichen Gesichtspunkten impliziert der deutsche Begriff der „Konfession“ bzw. „Konfessionslosigkeit“ zudem die Zustimmung bzw. Ablehnung des Bekenntnisses (confessio) einer Religionsgemeinschaft. Das Judentum, der Islam und der Buddhismus kennen zwar das "Shema Jsrael“ (Dtn 6, S. 4-9) sowie das Bekenntnis zu Allah als dem einen Gott und zu Muhammad als seinem Propheten (schahada) und die Zustimmung zur buddhistischen Lehre (dharma) als liturgische Handlungen, doch haben diese nicht die Funktion einer Bekenntnisgrundlage wie in den christlichen Konfessionen in Deutschland (vgl. Schröder, 2014). 
Die formalen und inhaltlichen Merkmale der Zugehörigkeit bzw. Nicht-Zugehörigkeit betreffen nicht nur das national verfasste Mitgliedschaftsrecht, sondern auch das transnationale, d. h. nicht an eine Nation gebundene Selbstverständnis der Religionsgemeinschaften. Im Judentum ist die Abstammung von einer jüdischen Mutter das zentrale Zugehörigkeitskriterium, d. h. ein Jude bzw. eine Jüdin kann sich prinzipiell nicht vom Judentum lösen, auch wenn er oder sie an keiner religiösen Praxis im Kontext einer Synagogengemeinde partizipiert und sogar das jüdische „Bekenntnis“, das „Shema Jsrael“ explizit ablehnen sollte. In Israel werden solche Personen „weltliche Juden" genannt (jehudim chiloniim). Ein förmliches Mitgliedschaftsrecht und eine Bekenntnisgrundlage nach christlichem Verständnis gibt es weder im Judentum noch im Staat Israel (vgl. Schröder, 2014, S. 224).

Im Islam wiederum begründet nicht die Abstammung von einer muslimischen Mutter die Zugehörigkeit zur Gemeinschaft der Gläubigen (umma), sondern das Aussprechen des Bekenntnisses zu Allah als dem einen Gott und zu Muhammad als seinem Propheten (schahada) vor drei Zeugen. Ein förmliches Mitgliedschaftsrecht gibt es auch hier nicht, damit auch nicht das Recht auf Austritt aus der Glaubensgemeinschaft (vgl. Schröder, 2014, S. 225).

Im Buddhismus gilt schließlich das Aussprechen der sog. dreifachen Zuflucht (trisharana): zu Buddha, zur Lehre (dharma), zur Mönchsgemeinde (sangha) und die damit einhergehende Selbstverpflichtung auf fünf sittliche Regeln (pancasila) als Zugehörigkeitserklärung. Insofern Zustimmung und Partizipation an einer religiösen Praxis über die Zugehörigkeit entscheiden, kennt auch der Buddhismus kein förmliches Mitgliedschaftsrecht (vgl. Schröder, 2014, S. 225).

Aufgrund der verschiedenen Verständnisse und Verfasstheiten kann der deutsche Begriff der Konfessionslosigkeit nicht ohne weiteres in andere nationale oder religiöse Kontexte übersetzt werden. Dieses terminologische Problem kennt auch der „Internationale Bund der Konfessionslosen und Atheisten e.V.“ (IBKA), der sich im Englischen „International League of Non-Religious and Atheists" und im Französischen "Ligue Internationale des Non-religieux et des Athées“ nennt (IBKA, 2020). Die Begriffe „non-denominational” und „non-confessional“ kamen für die Übersetzung nicht in Frage. Sie beziehen sich u.a. in den USA auf Kirchen, die keinem bestimmten Bekenntnis (z.B. der Confessio Augustana o.ä.), sondern allein der Bibel und dem Glauben verpflichtet sind, meinen also ziemlich genau das Gegenteil von dem, was der IBKA inhaltlich vertritt. Ins Französische lässt sich der Begriff der Konfessionslosigkeit nur durch das Kompositum „sans confession“ übersetzen, was dem Adjektiv „konfessionslos“ entspricht. Ein französisches Substantiv lässt sich aus dem Adjektiv aber nicht bilden. Da „confession“ häufig als Synonym für „Religion“ gebraucht wird, fühlt sich jemand, der „sans confession" ist, allgemein keiner Religion zugehörig. Konfessionen sind im französischen Sprachgebrauch sowohl die verschiedenen Religionen, als auch die verschiedenen Konfessionen im Sinne der Unterscheidung von katholisch, evangelisch und orthodox, sodass es die „confession musulmane“ oder „juive“ gibt (Französische Statistik-Seite, 2020). Als Teilgebiet der transnationalen Religions- und Bildungsforschung beschäftigen sich die Translation Studies (Weidlich, 2020) mit solchen Übersetzungsschwierigkeiten und den damit einhergehenden Herausforderungen interkultureller und interreligiöser Verständigung.

\section{Religionspädagogische Aspekte: Zur weiteren Erforschung religiöser Bildung in einer globalisierten Welt}

Als einer der größten nichtstaatlichen Bildungsanbieter in der Bundesrepublik Deutschland hat sich die Evangelische Kirche in Deutschland, bestehend aus einem Zusammenschluss von 20 Evangelischen Landeskirchen, in den vergangenen Jahren intensiv mit den wachsenden Aufgaben religiöser Bildung in einer globalisierten Welt beschäftigt. Im Kern geht es ihr darum, die globale Dimension des christlichen Glaubens stärker bewusst zu machen, die weltweite Vernetzung kirchlicher Bildungsakteure zu fördern und das transnationale Humankapital religiös gebildeter Menschen herauszustellen (vgl. Käbisch, 2018b)

Zuletzt hat sich die Kammer der EKD für Bildung und Erziehung, Kinder und Jugend intensiv mit den Aufgaben religionsbezogener Bildung angesichts wachsender Konfessionslosigkeit beschäftigt (EKD, 2020). Das Policy-Papier stellt u.a. die Pfadabhängigkeit der Entwicklungen in Deutschland heraus, da in anderen Ländern (vor allem außerhalb Europas) gänzlich andere Dynamiken im religiösen Feld zu beobachten sind. Dazu zählen u.a. „das rapide Anwachsen verschiedener christlicher Denominationen sowie des Islam in der südlichen Hemisphäre, die Ausprägung fundamentalistischer oder jedenfalls nicht von der westlichen Aufklärung affizierter Lesarten des christlichen Glaubens und anderer Religionen" (EKD, 2020, S. 18 f.). Auch wenn viele Schülerinnen und Schüler vor allem in Ostdeutschland in einer lokalen Kultur der Konfessionslosigkeit aufwachsen, in der die Nicht-Zugehörigkeit zu einer Kirche selbst schon zur Familientradition geworden ist und Religion keine unmittelbare Bedeutung für die eigene Lebensgestaltung zu haben scheint, kommen ihnen diese globalen Kontexte häufig sehr nahe: Sei es „durch Migration, durch mediale Berichterstattung, durch persönliche Begegnung im Rahmen wirtschaftlicher, kultureller, touristischer Kontaktaufnahme“ (EKD, 2020, S. 73).

Der vorliegende Beitrag ist nicht der Ort, um die daraus resultierenden Aufgaben religiöser Bildung in einer globalisierten Welt en détail auszuführen und auf Bildungsangebote für Konfessionslose zu beziehen. Gleichwohl sollen die abschließenden Stichpunkte deutlich machen, welches Tableau an Möglichkeiten das Grundlagenpapier der EKD beschreibt und welche Forschungsfragen sich daraus ergeben.

Die Thematisierung der globalen Dimension von Religion im schulischen Religionsunterricht ist die am einfachsten zu realisierende Möglichkeit, diese Dimension in schulischen Kontexten zur Geltung bringen, z.B. durch die Beschäftigung mit der weltweiten Übersetzungs- und Verbreitungsgeschichte des Neuen Testaments als ein "document of transnationalism“ (Käbisch, 2014; Käbisch, 2018a). Den konfessionellen Religionsunterricht besuchen nicht nur evangelische, römisch-katholische und muslimische Kinder und Jugendliche, sondern zu einem beträchtlichen Teil auch konfessionslose. Der Unterricht sollte daher so gestaltet sein, dass sich alle eingeladen fühlen 
und ins Gespräch kommen können. Unter welchen didaktischen Bedingungen der Dialog gelingen und die Pluralitätsfähigkeit der Schülerinnen und Schüler weiterentwickelt werden kann, ist bisher nur in Ansätzen untersucht worden.

Zur globalen Bildung gehört aber auch die Auseinandersetzung mit der Spannung zwischen lokalen und nationalen Gegebenheiten sowie den globalen und transnationalen Entwicklungen in Geschichte und Gegenwart. Nicht wenige Kommentatoren des Reformationsjubiläums 2017 haben u.a. kritisiert, dass der Anlass weitgehend ungenutzt geblieben sei, um die Reformation als europäisches und damit transnationales Ereignis zu feiern (z.B. Kaufmann, 2015). Exkursionen zu den nationalen, im 19. Jahrhundert entstandenen Lutherdenkmalen in Wittenberg, Eisleben und Eisenach, die das ikonische Vorbild für den hundertausendfach reproduzierten Playmobil-Luther lieferten, können daher ein Anlass sein, national verengte Geschichtsbilder zu problematisieren und die Reformation in einem europäischen oder transnationalen Zusammenhang zu thematisieren. Da die Kirchengeschichtsdidaktik kaum im Fokus der religionspädagogischen Forschung steht, ist deren Potential für das globale Lernen bislang kaum untersucht worden.

Internationale Jugendbegegnungen bieten konfessionslosen Schülerinnen und Schülern eine weitere Möglichkeit, transnationale Bildungsräume zu gestalten und die globale Dimension von Religion zu entdecken. Die unterschiedliche Rolle von Religion in anderen Ländern, die aufgezeigte Nichtübersetzbarkeit des deutschen Verständnisses von Konfessionslosigkeit und die unterschiedliche Stellung des Religionsunterrichts an staatlichen Schulen (z.B. in Frankreich, Deutschland und Polen) können Konfessionslose dazu anregen, über die Bedeutung von Religion im eigenen Land und in globalen Kontexten nachzudenken. Erste empirische Studien auf diesem Feld (vgl. Ilg, 2016, S. 322) sollten in Zukunft intensiviert werden.

Schulpartnerschaften mit Schulen auf anderen Kontinenten, wie etwa das über das EKD-finanzierte Projekt www. gpenreformation.net, das aus der Initiative www.schools500reformation.net hervorgegangen ist, sind ebenso dazu geeignet, mit Konfessionslosen den Welthorizont des christlichen Glaubens zu entdecken. Die konzeptionellen Beobachtungen und Erfahrungsberichte (vgl. Simojoki, Scheunpflug \& Kohlmann, 2016) sollten zum Anlass für eine stärkere empirische Erforschung von Schulpartnerschaften genommen werden.

Schließlich können, wie eingangs gezeigt, Sprachreisen und Schüleraustauschprogramme für konfessionslose Schülerinnen und Schüler einen Anlass bieten, sich mit der Bedeutung von Religion in globalen Kontexten zu beschäftigen. Die von einer Gastfamilie, wie etwa in den USA, erwartete Teilnahme am Gottesdienst und die damit einhergehende Erwartung, sich in den Alltag und die Kultur des Gastlandes zu integrieren, setzten mehr als nur konfessions- oder religionskundliches Wissen voraus. Es geht um Taktgefühl, Respekt und Toleranz gegenüber anderen Lebensentwürfen und fremden kulturellen Praktiken - also um die Mindeststandards, die eine zeitgemäße religiöse und religionsbezogene Bildung zu bieten hat.

\section{Literatur}

Asbrand, B. \& Scheunpflug, A. (2005). Zum Verhältnis von interreligiösem, interkulturellem, ökumenischem und globalem Lernen. In P. Schreiner, U. Sieg \& V Elsenbast (Hrsg.), Handbuch interreligiöses Lernen (S. 268-281), Gütersloh: Gütersloher Verlagshaus.

Bevölkerungsstatistik Frankreich (2020): Appartenance religieuse des jeunes de 18 a 30 ans en France en juin 2016. Zugriff am 04.07.2020 https://fr.statista.com/statistiques/600092/appartenance-religieuse-des-jeunes-france/

EKD (2020). Religiöse Bildung angesichts von Konfessionslosigkeit. Aufgaben und Chancen. Ein Grundlagentext der Kammer der EKD für Bildung und Erziehung, Kinder und Jugend. Leipzig: Evangelische Verlagsanstalt.

GLS Sprachenzentrum Berlin (2020). Zugriff am 04.07.2020 www.gls-sprachenzentrum.de/353_auslandsjahr_usa.html

IBKA (2020). Zugriff am 04.07.2020 www.ibka.org/infos/satzung.html

Ilg, W. (2016). Internationale Begegnungen im Kontext der Jugendarbeit. Empirische Ergebnisse aus einem unterschätzten Feld non-formaler Bildung. Zeitschrift für Pädagogik und Theologie, 68, 312-326. https://doi.org/10.1515/zpt-2016-0033

Käbisch, D. (2014). Religionsunterricht und Konfessionslosigkeit. Eine fachdidaktische Grundlegung. Tübingen: Mohr Siebeck.

Käbisch, D. (2018a). Bibel und Bibelübersetzungen in transnationaler Perspektive. In M. Zimmermann \& R. Zimmermann (Hrsg.), Handbuch Bibeldidaktik (S. 92 99). Tübingen: Mohr Siebeck.

Käbisch, D. (2018b). Religiöse Bildung als „transnationales Humankapital“? Soziologische, historische und religionspädagogische Aspekte. In H. Simojoki, A. Scheunpflug \& M. Schreiner (Hrsg.), Evangelische Schulen und religiöse Bildung in der Weltgesellschaft. Die Bamberger Barbara-Schadeberg-Vorlesungen (S. 51-68). Münster: Waxmann.

Käbisch, D. \& Simojoki, H. (2018). Säkularisierung, Globalisierung und die historische Pfadabhängigkeit des religiösen Wandels. Zur Verschränkung gesellschaftsund geschichtswissenschaftlicher Zugänge in der Religionspädagogik. In T. Heller (Hrsg.), Religion und Bildung - interdisziplinär (S. 271-291). Leipzig: Evangelische Verlagsanstalt.

Kaiser, J.-Chr. (1981). Arbeiterbewegung und organisierte Religionskritik. Proletarische Freidenkerverbände in Kaiserreich und Weimarer Republik. Stuttgart: KlettCotta.

Kaufmann, T. (2015). Europa und die Reformation. Frankfurter Allgemeine Zeitung, $248,6$.

Martin, D. (2011). East Germany: The World's Most Secular Society. In D. Martin (Hrsg.), The Future of Christianity. Reflections on Violence and Democracy, Religion and Secularization (S.149-164). Farnham: Ashgate. https://doi.org/10.1111/j.175 6-1183.2011.00115.x

Nowak, K. (1996). Staat ohne Kirche? Überlegungen zur Entkirchlichung der evangelischen Bevölkerung im Staatsgebiet der DDR. In G. Kaiser \& E. Frie (Hrsg.), Christen, Staat und Gesellschaft in der DDR (S. 23-43). Frankfurt a. M. u. New York: Campus.

Pickel, G. (2011). Atheistischer Osten und gläubiger Westen? Pfade der Konfessionslosigkeit im innerdeutschen Vergleich. In G. Pickel \& K. Sammet (Hrsg.), Religion und Religiosität im vereinigten Deutschland. Zwanzig Jahre nach dem Umbruch (S. 43-77). Wiesbaden: V\&S-Verlag. https://doi.org/10.1007/978-3-531-92649-0 $-3$

Schröder, B. (2014). Konfessionslosigkeit und religiöse Bildung in anderen Religionen und Ländern. Vergleichende Perspektiven. Zeitschrift für Pädagogik und Theologie, 66, 223-232. https://doi.org/10.1515/zpt-2014-0305

Simojoki, H. (2012). Globalisierte Religion. Ausgangspunkte, Maßstäbe und Perspektiven religiöser Bildung in der Weltgesellschaft. Tübingen: Mohr Siebeck.

Simojoki, H., Scheunpflug, A. \& Kohlmann, S. (2016). Schulentwicklung und religiöses Lernen im transnationalen Horizont. Gestaltungsperspektiven am Beispiel des Projekts „schools500reformation“. Zeitschrift für Pädagogik und Theologie, 68, 327-339. https://doi.org/10.1515/zpt-2016-0034

Weidlich, L. (2020), Art. Translation Studies. In Wissenschaftlich Religionspädagogisches Lexikon im Internet. Zugriff am 11.07.2020 www.wirelex.de

\section{Dr. David Käbisch}

Professor, Studium der Evangelischen Theologie für das Pfarramt (Erste Kirchliche Examen) und das Lehramt an höheren Schulen (Erste und Zweite Staatsexamen) in Leipzig, Jerusalem (Israel) und Cheltenham (England); 2008 Promotion zum Dr. theol. in Jena; 2013 Habilitation im Fach Praktischen Theologie in Marburg; seit 2013 Inhaber der Professur für evangelische Religionspädagogik an der Goethe-Universität in Frankfurt am Main. Seine Forschungsschwerpunkte sind religionsbezogene Bildung in ökumenischen, interreligiösen und konfessionslosen Kontexten sowie die transnationalen Dimensionen religiöser Bildung in der Moderne. 